Institutions

Markets

Technologies

IMT

INSTITUTE

FOR ADVANCED

STUDIES

LUCCA

\title{
An Extreme Value Analysis of the Last Century Crises Across Industries in the U.S. Economy
}

Marco Bee

Massimo Riccaboni

Luca Trapin 
ISSN 2279-6894

IMT LUCCA EIC WORKING PAPER SERIES \#02/2016

(C) IMT Institute for Advanced Studies Lucca

Piazza San Ponziano 6, 55100 Lucca

Research Area

Economics and institutional change

\section{An Extreme Value Analysis of the Last Century Crises Across Industries in the U.S. Economy}

\section{Marco Bee}

Department of Economics and Management, University of Trento

\section{Massimo Riccaboni}

IMT Institute for Advanced Studies Lucca;

Department of Managerial Economics, Strategy and Innovation, K.U. Leuven

\section{Luca Trapin}

IMT Institute for Advanced Studies Lucca 


\title{
An Extreme Value Analysis of the Last Century Crises Across Industries in the U.S. Economy
}

\author{
Marco BEE* $\quad$ Massimo RICCABONI ${ }^{\dagger} \quad$ Luca TRAPIN ${ }^{\ddagger}$
}

February 19, 2016

\begin{abstract}
The two large scale crises that hit the world economy in the last century, i.e. the Great Depression and the Great Recession, have similar outbreak and recovery patterns with respect to several macroeconomic variables. In particular, the largest depressions are likely to be accompanied by stock-market crashes. This study investigates the behavior of the U.S. stock market before, during and after deep downturns, focusing particularly on the tails of the return distribution. We develop two automatic procedures to identify multiple change-points in the tail of financial time series as well as in the co-crash and co-boom probabilities of different markets. We then apply our methodology to twelve time series representative of the sectors of the U.S. economy. We find that regime shifts in the lower tail of the distribution tend to co-occur before deep downturns. Our results contribute to a better understanding of the origin and systemic nature of large scale events to make policy interventions more timely and effective.
\end{abstract}

Keywords: Financial Crisis, Extreme Value Theory, Change-points Detection, U.S. Economy. JEL Classification: C1, G01.

\footnotetext{
${ }^{*}$ Department of Economics and Management, University of Trento, Trento, Italy

${ }^{\dagger}$ IMT Institute for Advanced Studies, Lucca, Italy and Department of Managerial Economics, Strategy and Innovation, K.U. Leuven, Leuven, Belgium.

${ }^{\ddagger}$ IMT Institute for Advanced Studies, Lucca, Italy
} 


\section{Introduction}

"September and October of 2008 was the worst financial crisis in global history, including the Great Depression."

-Ben Bernanke

The long economic downturn that began in late 2007 and is known as the Great Recession has raised new concerns regarding the possibility of a depression period (Eggertsson and Mehrotra, 2014). This has produced a widespread debate about the differences and commonalities of these events and those experienced during the Great Depression of the 1930s (Bordo and James, 2010; Stiglitz, 2015).

Both crises started in the United States, but had huge effects on the economy worldwide, forcing national governments to extraordinary commitments. The Great Depression lies its roots in the collapse of the stock market. The market exuberance of the mid-twenties prompted people to borrow money and investing in stocks. This created a spurge in the stock prices that culminated in the stock market collapse of the 23rd October 1929. The losses suffered by the investors made them unable to repay their debts causing the default of a large number of banks. The Great Recession is characterized by a similar scenario, but the housing market was the playground in this case. People were buying houses with sub-prime mortgages bearing high interest rates but guaranteed by the value of the house itself. The fall of the houses price coupled with the inability of creditors to meet their obligations caused large losses in the banking sector and among institutional investors.

Even though the origins are different, the Great Depression and the Great Recession are similar with respect to the evolution of several macroeconomic variables, such as the decline in GDP, investment and consumption and the rise of the unemployment rate (Bordo and James, 2010). As noticed by Barro and Ursúa (2009), stock market crashes provide important guidance about the prospects for depression. They find, for 25 countries with long-term data, that stock market crashes go along with minor depressions (multi-year decline of consumption 
or GDP of 10\%) 30\% of the time and major depressions (declines by more than 25\%) $11 \%$ of the time. Therefore, structural changes in the dynamics of the stock market can be used as an early-warning signal of macroeconomic contraction. In particular, given the evidence of heavy tails for the stock return distribution (Mandelbrot, 1963; Fama, 1965), structural changes of the tail probabilities are important because they entail changes in the likelihood of observing extreme events. In this paper, we study the tails of the unconditional return distribution.

Change-point detection in the tails has attracted a great deal of attention in the last decade. Early studies of Quintos et al. (2001) report evidence of changes in the tail heaviness for three Asian countries during the Asian financial crises of the mid-nineties. Candelon and Straetmans (2006) extend the test of Quintos et al. (2001) to multiple change-points and apply it to Asian and Western exchange rate data to assess the impact of monetary policies. A different but related approach has been used in Bee et al. (2015) to uncover tail seasonality in several U.S. industries. Straetmans et al. (2008) generalize these frameworks to the bivariate joint distribution and propose to test changes in the co-crash probability (joint lower tail) and co-boom probability (joint upper tail) between two assets. This test can be used to understand changes in the probability of contagion or joint crashes.

We rely on these techniques to investigate whether the tails of the unconditional return distribution of twelve financial time series providing a market proxy of the U.S. economic sectors behaved differently during the Great Recession and the Great depression. The availability of stock market data for almost a century allows for an integrated analysis of the two crises that would not be otherwise possible with standard long-run economic data. We develop a new algorithm based on the test of Kim et al. (2009) to detect and estimate multiple change-points in the tails of the time series of the U.S. economic sectors over the last century. This allows us to evaluate which sectors were more prone to extreme events during the two crises and the diffusion pattern of regime shifts in the negative tail of returns across sectors. In the spirit of Straetmans et al. (2008), we then extend our methodological approach to 
the multivariate framework to shed more light on the contagion pattern of the crisis. We perform a bivariate analysis aimed at identifying multiple change-points in the co-crash and co-boom probabilities across sectors. As a consequence, we can evaluate tail spillovers and contagion, along with the implications for systemic risk.

Both the univariate and bivariate approaches rely on an algorithm for multiple changepoint detection. Therefore, it is not necessary to specify in advance the dates of the tail structural breaks. This constitutes an advantage as we let the data speak for themselves without interfering with the statistical properties of the time series. We find several changepoints in both the upper and lower tails of the different sectors and changes in the co-crash and co-boom probabilities. All the structural breaks can be justified on strong economical basis as they correspond to the main economic events that characterized the nineteenth century and the first decade of the new millennium. We focus our discussion on the changepoints close to the Great Depression and the Great Recession in order to see how the two crises affected the economy and whether the policy responses were effective.

Our main findings can be summarized as follows. First, all the economic sectors are affected by the two crises, both at the univariate level, with an increase of extreme events, and at the multivariate level, with an increase in the probability of co-crash and systemic risk. Second, the change-points tend to anticipate the crucial dates of the two crises, so that the detection of tail change points can be used as early warnings for large scale financial crisis. Change-points in the financial sector occur earlier than in the others in both crises, consistently with the fact that the banking industry was at the core of the crises' origins. Finally, the recovery time, defined as the time elapsed before another change-point occurs, is different in the two cases, and we suggest to relate it to the effectiveness of the policies implemented by the US government.

The remainder of the paper is structured as follows. Section 2 presents the econometric model and the change-point algorithms. Section 3 illustrates the results of the univariate and bivariate empirical analyses. Finally, Section 5 concludes. 


\section{Methodology}

As our analysis covers both the univariate and bivariate case, we first define the econometric model describing the dynamic of the assets considered, i.e. the economic sectors. We then describe the strategy used to identify multiple change-points in the upper and lower tails of a single asset and in the co-crash and co-boom probabilities of two assets.

\section{$2.1 \quad$ Econometric model}

Let $p_{j, t}$ be the logarithmic price of the $j$-th asset at time $t$, with $j \in\{1, \ldots, K\}$. Assume that the marginal distribution of the $j$-th daily log-return $r_{j, t}=p_{j, t}-p_{j, t-1}$ follows a GARCH process (Bollerslev, 1986):

$$
\begin{aligned}
r_{j, t} & =\sigma_{j, t} \epsilon_{j, t}, \\
\sigma_{j, t}^{2} & =\omega_{j}+\alpha_{j} \epsilon_{j, t-1}^{2}+\beta_{j} \sigma_{j, t-1}^{2},
\end{aligned}
$$

with dependence structure defined by $\boldsymbol{\epsilon}_{\boldsymbol{t}}=\left[\epsilon_{1, t}, \ldots, \epsilon_{K, t}\right] \sim$ iid $F$. In what follows, we drop the subscript $j$ and simply refer to $r_{t}$ for convenience, unless this comes at the expense of clarity.

Carrasco and Chen (2002) prove that the process in Equation (1) satisfies the $\beta$-mixing condition

$$
\beta(l)=\sup _{m} \mathbb{E}\left\{\sup _{A \in \mathcal{F}_{m+l+1}^{\infty}}\left|P\left(A \mid \mathcal{F}_{1}^{m}\right)-P(A)\right|\right\} \stackrel{l \rightarrow \infty}{\longrightarrow} 0,
$$

with $\mathcal{F}_{l}^{m}=\mathcal{E}\left\{r_{t}: t=l, \ldots, m\right\}, \mathcal{F}_{l}^{\infty}=\mathcal{E}\left\{r_{t}: t=m, m+1, \ldots\right\}$ and $\mathcal{E}\{\cdot\}$ denoting the information set. Mikosch and Starica (2000) prove that the marginal distribution of $r_{t}$ is regularly varying, thus it presents power-law tail behavior with tail index $1 / \xi$ :

$$
P\left(r_{t}>x\right)=\mathcal{L}(x) x^{-1 / \xi} \text { as } x \rightarrow \infty,
$$


with $\mathcal{L}(x)$ a slowly varying function, i.e. such that $\mathcal{L}(d x) / \mathcal{L}(x) \rightarrow 1$ as $x \rightarrow \infty$ with $d$ constant.

\subsection{Tail change-points identification}

Let the sequence $\left\{r_{t}\right\}_{t=1}^{n}$ be a sequence following the process described in Equation (1). To identify a structural change in the tail index $\xi$ we consider the hypotheses

$$
\mathcal{H}_{0}: \xi_{1}, \ldots, \xi_{n}=\xi \quad \mathcal{H}_{1}: \operatorname{Not} \mathcal{H}_{0}
$$

Note that one can investigate both tails of $r_{t}$ over time by looking for changes in the tail index of the sequence $r_{t}$ and the negated sequence $-r_{t}$, respectively. To test the hypothesis (4), Kim et al. (2009) suggest the following cusum-type statistic

$$
T_{n}=\frac{1}{\sqrt{n \tau}} \max _{\lambda n \leq l \leq(1-\lambda) n}\left|\sum_{t=1}^{l} I\left(r_{t}>q_{\tau}\right)-\frac{l}{n} \sum_{t=1}^{n} I\left(r_{t}>q_{\tau}\right),\right|
$$

where $I(\cdot)$ is the indicator function, $q_{\tau}$ is the $\tau$ th sample quantile of $\left\{r_{t}\right\}$ with $\tau$ close to one, $l$ are the candidate change-points, and $\lambda$ is set equal to a small positive value to prevent intervals becoming too small. Assuming that conditions (2) and (3) hold, Kim et al. (2009) show that under $\mathcal{H}_{0}$

$$
\frac{1}{\sqrt{(1+\omega)}} T_{n} \stackrel{n \rightarrow \infty}{\longrightarrow} \mathcal{B}
$$

where $\mathcal{B}$ is the distribution of $\sup _{0 \leq t \leq 1}|B(t)|$ with $B(t)$ a Brownian Bridge and $\omega$ is a parameter that corrects the test for the dependence in the time series of returns (Quintos et al., 2001).

To identify multiple change-points in the return sequence, we implement a new sequential strategy that returns both the optimal number $m$ of change-points and their locations $\hat{l}_{1}, \ldots, \hat{l}_{m}$. First, we carry out the test in equation (5) over the whole sequence $r_{t}$. If $T_{n}<\mathcal{B}_{\alpha}$, where $\mathcal{B}_{\alpha}$ is the (1- $\left.\alpha\right)$-quantile of $\mathcal{B}$, then there is no change-point and the 
procedure terminates. Otherwise, $\mathcal{H}_{0}$ is rejected and the first change-point is set to be $\hat{l}_{1}=\arg \max _{l}\left|\sum_{t=1}^{l} I\left(r_{t}>q_{\tau}\right)-\frac{l}{n} \sum_{t=1}^{n} I\left(r_{t}>q_{\tau}\right)\right|$. Multiple change-points are obtained performing the test in Equation (5) iteratively over the sub-intervals of length $s$ defined by the change-points identified at the $(m-1)$ th iteration on $r_{t}$, and setting the $m$ th changepoint equal to $\hat{l}_{m}=\arg \max _{l}\left|\sum_{t=1}^{l} I\left(r_{t}>q_{\tau}\right)-\frac{l}{s} \sum_{t=1}^{s} I\left(r_{t}>q_{\tau}\right)\right|$, if $\mathcal{H}_{0}$ is rejected at the level $\alpha /(m+1)$. This iterative procedure continues until the test fails to reject the null in each segment, or when the shortest segment defined by adding another change-point is shorter than $\lambda n$.

Once the change-points are identified, we can study the different tail regimes using the peaks-over-thresholds method (Embrechts et al., 1997). Let $\left\{X_{t}\right\}$ be a sequence of iid observations with marginal distribution $F$ endowed with upper end point $v_{F}:=\sup \left\{\eta_{t}: F\left(\eta_{t}\right)<\right.$ 1\}. Given a high threshold $u, u<v_{F}$, Pickands (1975) shows that when $u \rightarrow v_{F}$, the distribution of the excesses $\left(X_{t}-u\right)_{+}$converges to a Generalized Pareto (GP) distribution $G$ with shape parameter $\xi$ and scale parameter $\nu>0$. That is, $P\left(X_{t}-u \leq x \mid X_{t}>u\right)$ goes to

$$
G(x ; \xi, \nu)= \begin{cases}1-\{1+\xi x / \nu\}^{-\frac{1}{\xi}} & \text { for } \quad \xi \neq 0 \\ 1-\exp \{-x / \nu\} & \text { for } \quad \xi=0\end{cases}
$$

as $u \rightarrow v_{F}$. When $\xi>0, F$ has Pareto-type upper tail with tail index $1 / \xi$ corresponding to the power-law behavior of Equation 3 .

For each tail regime, corresponding to an interval defined by the change-points, the excesses $w_{t}=\left(r_{t}-u\right)_{+}$over a high threshold $u$ are identified, and the parameters $(\xi, \nu)$ of the GP distribution are estimated by Maximum Likelihood (ML). Note that the sequence $\left\{r_{t}\right\}$ is not independent, as it follows a GARCH process. Under the $\beta$-mixing condition of Equation (2) and some mild assumptions on the slowly varying function in Equation (3), Drees (2000) prove the consistency and asymptotic normality of the ML estimator of the GP distribution.

In the empirical analysis, we will consider a threshold level $u$ and a quantile $q_{\tau}$ corre- 
sponding to the 95th quantile of the time series considered, unless otherwise stated. Note that we will use the return time series $r_{t}$ to identify change-points in the upper tail and the negated return series $-r_{t}$ to identify change-points in the lower tail.

\subsection{Co-crash and co-boom change-points identification}

Following the intuition of Straetmans et al. (2008), we extend the change-points identification procedure presented in Section 2.2 to the bivariate framework. In contrast to Straetmans et al. (2008), when applied to the joint lower and joint upper tails, this approach allows us to identify change-points in the co-crash and co-boom probabilities automatically, without the need of specifying exogenously the date of the structural break.

Let $\left\{r_{1, t}\right\}_{t=1}^{n}$ and $\left\{r_{2, t}\right\}_{t=1}^{n}$ be the returns at time $t$ for two assets with joint distribution $G_{1,2}(\cdot, \cdot)$. We define the co-boom probability as

$$
\tau_{q}^{U P}=P\left(r_{1, t}>q, r_{2, t}>q\right)=\bar{G}_{1,2}(q, q),
$$

for a high quantile level $q$ such that $\bar{G}_{1,2}(q, q)$ is close to zero, where $\bar{G}(\cdot, \cdot)$ is the countercumulative distribution function. Note that an analogous definition of the co-crash probability $\tau_{q}^{L O W}$ can be obtained substituting $r_{t}$ with the negated returns $-r_{t}$ and inverting the dependence structure $G_{1,2}(\cdot, \cdot)$. The discussion that follows equally applies to both probabilities so we will generally discard the marks $U P$ and $L O W$.

In order to estimate the co-crash and co-boom probabilities, we rely on the semi-parametric approach devised by Ledford and Tawn (1996). The method consists in applying the probability integral transform to the series so as to obtain stationary unit-Fréchet series,

$$
y_{1, t}=-\frac{1}{\log \left[\widehat{G}_{1}\left(r_{1, t}\right)\right]}, \quad y_{2, t}=-\frac{1}{\log \left[\widehat{G}_{2}\left(r_{2, t}\right)\right]},
$$

where $\widehat{G}_{1}$ and $\widehat{G}_{2}$ are the empirical distribution functions. Next define the sequence of 
pairwise minima $\left\{y_{\min , t}\right\}_{t=1}^{n}$ as $y_{\min , t}=\min \left(y_{1, t}, y_{2, t}\right)$. As $y_{1, t}$ and $y_{2, t}$ are Fréchet, $y_{\min }$ is regularly varying, i.e.

$$
P\left(y_{\min }>x\right)=\mathcal{L}(x) x^{-1 / \eta}
$$

with $\mathcal{L}(x)$ a slowly varying function. This brings us back to the univariate framework of Equation (3), but now the tail index $1 / \eta$ measures not only the tail thickness of $y_{\min }$ but also the dependence of the pair $\left(r_{1, t}, r_{2, t}\right)$ in the tail region $[q, \infty) \times[q, \infty)$. The higher the value of $\eta$, the higher the probability of a co-crash or a co-boom.

To identify multiple change-points, we resort to the algorithm defined for the univariate framework in Section 2.2. Therefore we use the test statistics (5) to test the hypothesis

$$
\mathcal{H}_{0}: \eta_{1}, \ldots, \eta_{n}=\eta, \quad \mathcal{H}_{1}: \operatorname{Not} \mathcal{H}_{0}
$$

Note that, as a result of the transformation of the original series to unit-Fréchet, $y_{\min , t}$ does not have a GARCH structure anymore. In particular, there is no dependence in the first moment of the original series, and the possible dependence between the squares vanishes because of both the unit-Fréchet transformation and the fact that we are working with pairwise minima. Consequently, we simply treat the observations as independent, so that under $\mathcal{H}_{0}$, according to $(6)$,

$$
T_{n} \stackrel{n \rightarrow \infty}{\longrightarrow} \mathcal{B}
$$

where $\mathcal{B}$ is the distribution of $\sup _{0 \leq t \leq 1}|B(t)|$ with $B(t)$ a Brownian Bridge.

Once the change-points are identified, we can study the different regimes of the co-crash and co-boom probabilities estimating the parameter $\eta$ with the Hill (1975) estimator. We will refer throughout to $\eta^{U P}$ and $\eta^{L O W}$ respectively as the co-boom parameter and the co-crash parameter. 


\section{A comparison of the Great Depression and the Great Recession}

\subsection{An historical review}

The nineteenth century has been the stage of multiple stock market crashes, but none rivals the size and magnitude of the stock market collapse occurred on October 23, 1929. As early as in 1924, the Dow Jones Industrial Average started a long positive trend arriving at quadrupling its value in 1929. The exuberance of the market attracted investors chasing profits investing on margin with borrowed money. During this time, $40 \%$ of bank loans were used in order to purchase stocks. On October 23rd, 1929 the stock prices suddenly plummeted. Although the investors holding stocks were greatly affected, the segment that quite possibly was most hit was the banking sector.

The 2000 era opened with the worst global economic crisis since the Great Depression, the Great Recession, that grounds its foundation in the housing market crash. The causes of this collapse can be ascribed to the proliferation of sub-prime mortgages to individuals with low creditworthiness and the spread of structured products such as asset-backed and mortgage-backed securities among investors. The creditors' insolvency forced several banks to absorb billions of dollars in losses. Lehman Brothers went bankrupt and other large financial institutions such as Merrill Lynch, AIG, Freddie Mae and Fannie Mac came very close to following suit.

Both crises were characterized by a substantial decrease in GPD, however the magnitude of the decline was higher during the Great Depression. From 1929 to 1933 the real GDP fell in the U.S. by close to 30 percent, whereas between 2007-2009 it fell by a little over 5 percent (Bordo and James, 2010). Similarly, the unemployment rate in the U.S. peaked at 25 percent in 1933 versus a little above 10 percent in 2009. Although the two crises present similar characteristics, the policies implemented to guarantee the recovery are different. During the Great Depression, financial markets presented a low degree of regulation and 
this led to the release of important acts with the aim of reforming the banking sector and to the establishment of the Securities and Exchange Commission (SEC). At the same time, to stimulate the economy, President Roosevelt delivered a series of programs that are now known under the name of New Deal. During the Great Recession, the U.S. government heavily relied on fiscal policy to influence the economy and established an asset purchasing program fron financial institutions called TARP to strength the financial sector.

\subsection{Data description}

Before applying the statistical procedures presented in Section 2, we describe the features of data. As a market-based proxy of the U.S. economics sectors, we consider the daily returns of 12 equally-weighted industry portfolios available in the Kenneth R. French data library. The dataset contains records from 1923 to 2013 and has been created from the CRSP database, assigning each NYSE, AMEX and NASDAQ stock to an industry portfolio based on its four-digit SIC code. We consider an equally-weighted portfolio, but it would have been possible to use a value-weighted portfolio. We prefer to stick to the former because we aim at capturing a sector-wide behavior, and giving too much weight to highly capitalized firms might result in a portfolio with too much idiosyncratic risk. However, an unreported analysis of the value-weighted portfolios reveals that the change-points are qualitatively the same, especially those occurring in the proximity of the Great Depression and the Great Recession.

The sectors considered are listed in Table 1 along with summary statistics. The means and standard deviations are comparable to those observed in other studies. The skewness and kurtosis coefficients suggest a substantial degree of asymmetry and lepto-kurtosis in the shape of the distribution of these returns. The tail index for both the tails of the unconditional return distribution are positive across the sectors, consistently with the theoretical model put forth in Section 2.1. 
Table 1: Data description. For each sector, we report the abbreviation (Abbrv.), the empirical estimates of the mean, the standard deviation (Std. dev.), the skewness (Skew.) and the kurtosis (Kurt.), and the ML estimates of the tail parameter $\xi$ obtained by fitting the GP distribution above the 99th quantile of the upper $\left(\xi_{U P}\right)$ and the lower tail $\left(\xi_{L O W}\right)$.

\begin{tabular}{lccccccc}
\hline Sectors & Abbrv. & Mean & Std. dev. & Skew. & Kurt. & $\xi_{U P}$ & $\xi_{L O W}$ \\
\hline Business Equipment & BusEq & 0.09 & 1.38 & 0.18 & 23.55 & 0.18 & 0.17 \\
Chemicals & Chems & 0.08 & 1.15 & 0.07 & 31.61 & 0.25 & 0.22 \\
Durable & Durbl & 0.07 & 1.37 & 0.38 & 24.89 & 0.21 & 0.19 \\
Energy & Enrgy & 0.10 & 1.41 & 0.54 & 26.06 & 0.25 & 0.22 \\
Health & Hlth & 0.08 & 1.14 & 0.78 & 34.35 & 0.26 & 0.19 \\
Manufacturing & Manuf & 0.08 & 1.21 & 0.40 & 28.96 & 0.19 & 0.22 \\
Money & Money & 0.09 & 1.13 & 1.02 & 33.72 & 0.25 & 0.21 \\
No Durable & NoDur & 0.08 & 0.94 & 0.57 & 35.43 & 0.23 & 0.16 \\
Other & Other & 0.11 & 1.23 & 0.48 & 21.01 & 0.16 & 0.12 \\
Shops & Shops & 0.08 & 1.04 & 0.25 & 23.95 & 0.20 & 0.17 \\
Telecommunications & Telcm & 0.07 & 1.19 & -0.01 & 10.83 & 0.17 & 0.10 \\
Utilities & Util & 0.07 & 1.21 & 1.22 & 34.03 & 0.20 & 0.10 \\
\hline
\end{tabular}

\subsection{Tail change-points}

Figures 1 and 2 report the change-points obtained on the time series of the different economic sectors, respectively for the upper and lower tails. The two panels highlight that the changepoints tend to occur in a neighborhood of the main events that characterize the last century. One can notice the Great Depression, the recession period caused by the tightening of the fiscal policy after the New Deal at the end of the thirties, the energy crises of the seventies, the dot-com bubble, the Asian financial crises and the Great Recession. According to Figure 3 , it is possible to note that the two crises of interest here are those exhibiting a wider shock across the sectors, confirming the strong impact they had on the economy.

As our aim consists in studying the market behavior during the Great Depression and the Great Recession, we focus our attention on the change-points occurring in these periods. The key dates used as a reference point for the two crises are the 29th October 1929, known as the Black Tuesday, and the 11th October 2007, when the stock market reaches its maximum before falling for the subsequent two years. Tables 2 and 3 report the change-points closest to these crucial dates, respectively for the upper and the lower tail. We should be able to 
identify a first change-point where the tails become heavier as a consequence of the shock of the crisis and a second change-point signaling a more quiet period following the government policies.

The first remark is that the change-points in the lower tails tend to anticipate those in the upper tails in both crises. In particular, the Pre-crisis change-points in the lower tails occur before the 29th October 1929 for the Great Depression and before the 11th October 2007 for the Great Recession, while most of the Pre-crisis change-points in the upper tails occur after these dates. This signals that the market anticipated the possibility of a big shock on the market, thus leading to a change point in the lower tails. After the crises hit the economy, the volatility increased in the markets and bounce effects led to changes in the upper tail.

To appreciate the severity of the two crises, tables 4 and 5 display the values of the estimated parameters of the GP distribution fitted to the intervals preceding and following the Pre-crisis change point. This allows us to study the change in the tail behavior before and after the first structural break. Looking at the values of $\nu$, the effect of the crises on both tails and across all sectors is compelling. Less obvious is the effect on the tail parameter $\xi$, but this estimate tends to suffer of instability given the large sample size typically required for reliable inference. However, it is surely interesting to note that the Post-shock estimated values of $\xi$ for the Great Depression tend to be strongly positive, in line with the estimates obtained on the whole series and reported in Table 1. Contrarily, the Post-shock estimated values of $\xi$ for the Great Recession are close to zero, suggesting an exponential tail decay.

Comparing the Pre-crisis lower tail change-points of the different sectors, we can study cross-sectoral differences of the two crises when they hit the economy. According to Table 3, there seems to be a homogeneous timing among most of the sectors in the '29 crisis, while this pattern is less clear in the recent crisis. The first sectors to be hit are Telecommunications, Chemicals and Money in the Great Depression, and Money and Utilities in the Great Recession. This evidence on the Money sector is consistent with the role that banks and 
financial institutions had in both crises, as argued in the historical review of Section 3.1. The relevance of the Money sector is confirmed by the results in Figure 4: this sector is the one experiencing the highest number of tail change-points in the last century.

As to the difference between the two crises, we have already noticed that the pattern of diffusion was not the same, apart for the Money sector. The Telecommunication sector was among the first to be hit in '29, but one of the last in the recent crisis. Furthermore, the Health, Business Equipment and Energy sectors were not interested during the Great Recession. Another notable difference is in the recovery time of the stock market. From Table 3 it is evident that most of the Post-crisis change points in the Great Depression occurred five years after the Pre-crisis change-points, while in the Great Recession for most sectors the Post-crisis change-points occurred two-years after the Pre-crisis change-points. This can be interpreted as an index of efficiency of the policy responses implemented by the government. The fiscal policy carried over by the U.S. government in the 2008-2009 was a large intervention with a substantial and immediate impact on the economy. Contrarily, the interventions of the thirties were mostly directed to establish a suitable environment for the transactions in the stock market.

Table 2: Upper tail change-points dates.

\begin{tabular}{lccccc}
\hline \multirow{2}{*}{ Sectors } & \multicolumn{2}{c}{ Great Depression } & & \multicolumn{2}{c}{ Great Recession } \\
\cline { 2 - 3 } Business Equipment & Pre-crisis & Post-crisis & & Pre-crisis & Post-crisis \\
Chemicals & $1930-09-30$ & $1934-01-24$ & & $2008-09-17$ & - \\
Durable & $1929-02-08$ & $1931-06-02$ & & $2007-11-27$ & - \\
Energy & $1930-09-30$ & $1934-03-02$ & & $2007-11-27$ & - \\
Health & $1930-11-12$ & $1934-01-15$ & & $2008-03-10$ & - \\
Manufacturing & $1930-07-15$ & $1934-03-02$ & & $2008-07-07$ & - \\
Money & $1930-09-30$ & $1934-08-22$ & & $2007-08-16$ & - \\
No Durable & $1930-11-12$ & $1934-01-30$ & $2007-08-03$ & - \\
Other & $1930-09-30$ & $1934-03-02$ & & $2007-09-17$ & - \\
Shops & $1930-12-16$ & $1934-03-02$ & & $2007-11-27$ & - \\
Telecommunications & $1931-09-22$ & $1933-08-17$ & $2008-03-10$ & $2012-01-03$ \\
Utilities & $1929-01-28$ & $1931-04-25$ & & $2007-06-26$ & $2009-07-23$ \\
\hline
\end{tabular}


Figure 1: Upper tail change-points. Time line for each sector (lines) and identified change-points (red crosses). The level of the upper-tail quantile $q_{\tau}$ in the algorithm is $\tau=0.95$.

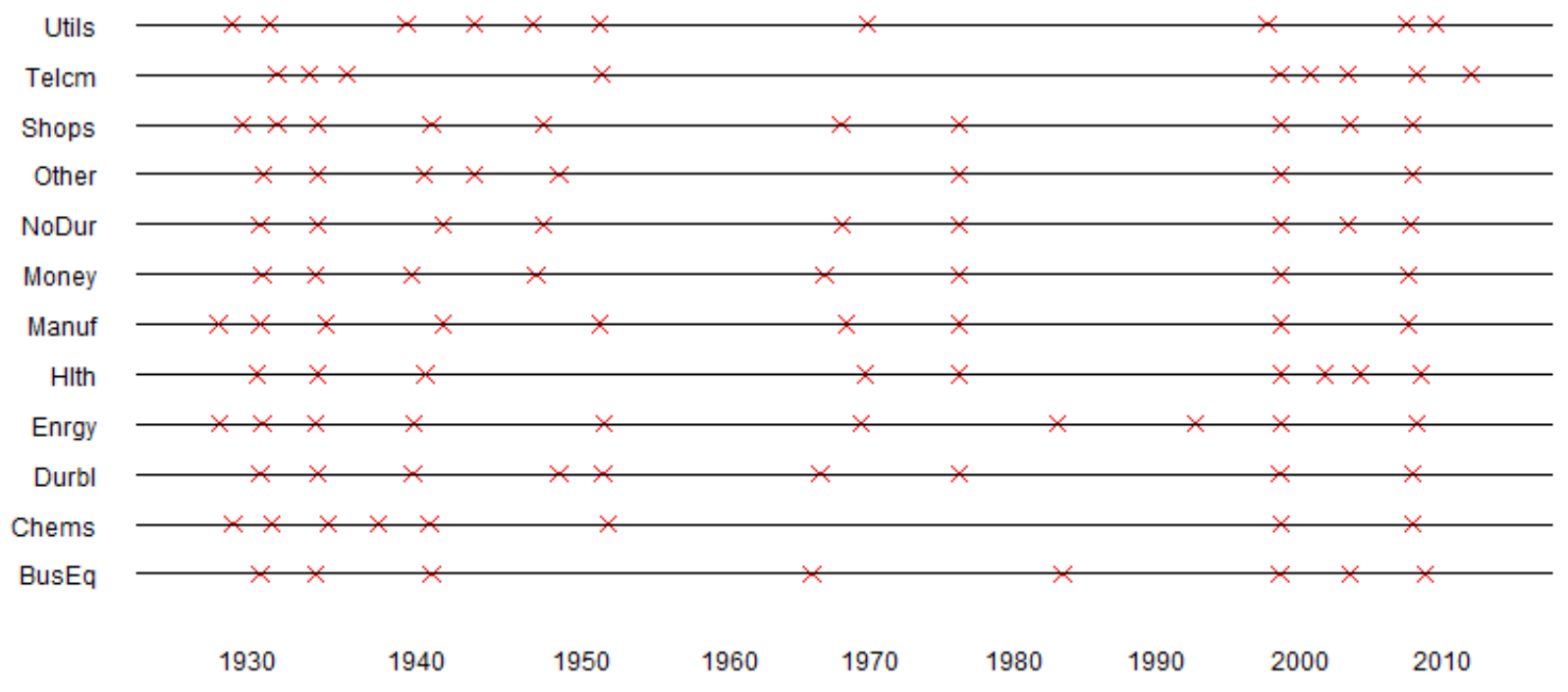

Figure 2: Lower tail change-points. Time line for each sector (lines) and identified changepoints (red crosses). The level of the lower-tail quantile $q_{\tau}$ in the algorithm is $\tau=0.95$.

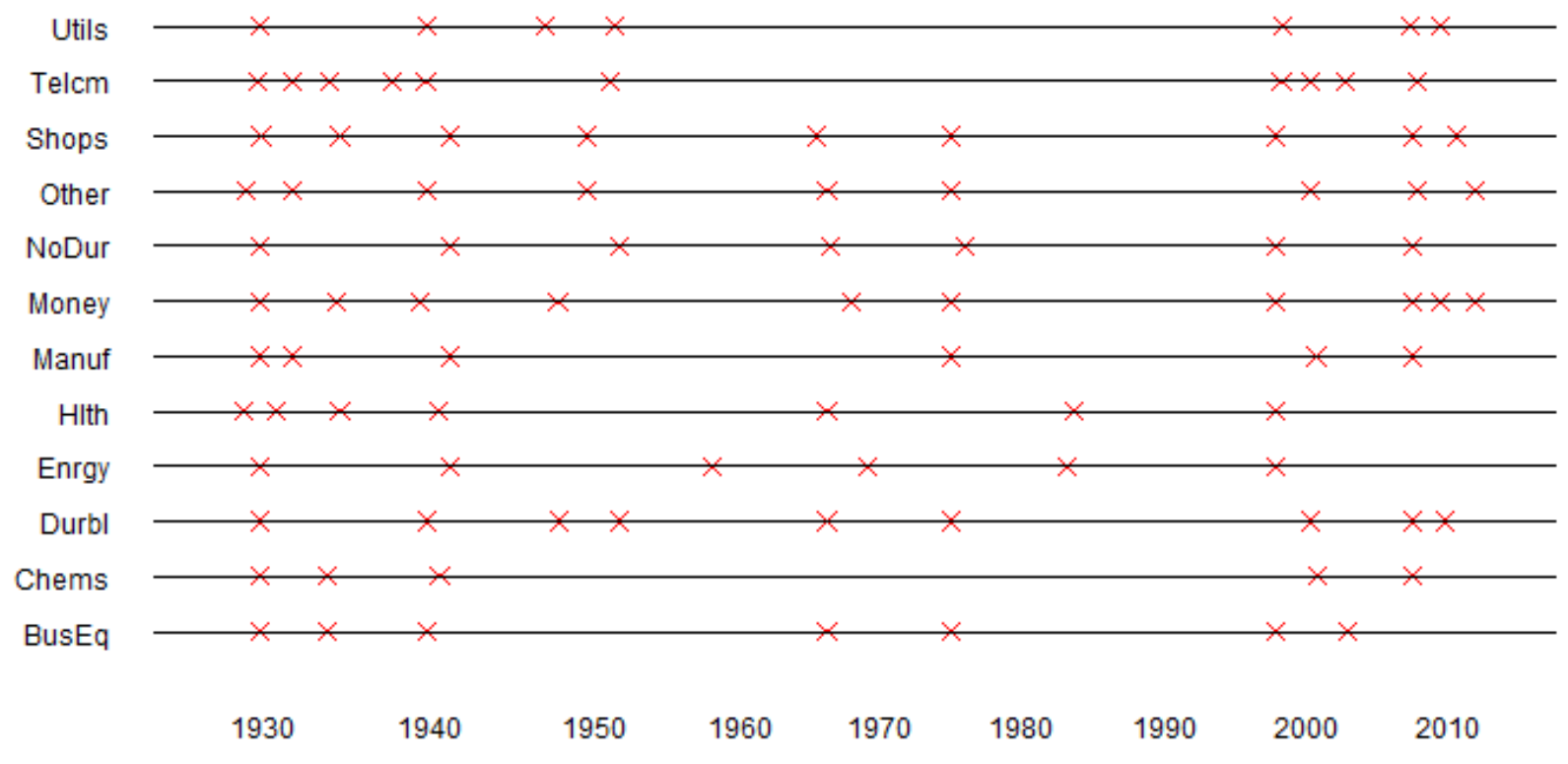


Figure 3: Number of tail change-points. Number of sectors ( $x$ axis) experiencing a change-point in the lower tail in a given year ( $y$ axis).

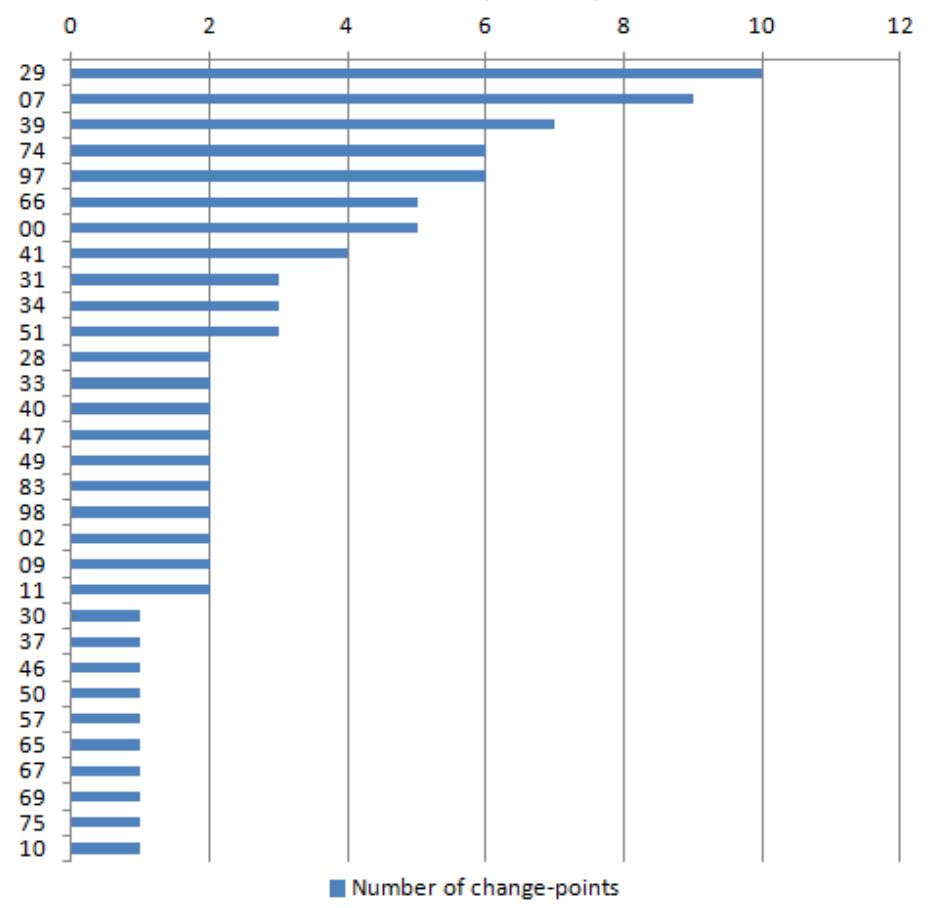

Figure 4: Sector tail change-points. Number of change-points ( $x$ axis) experienced by each sector ( $y$ axis) on the whole period.

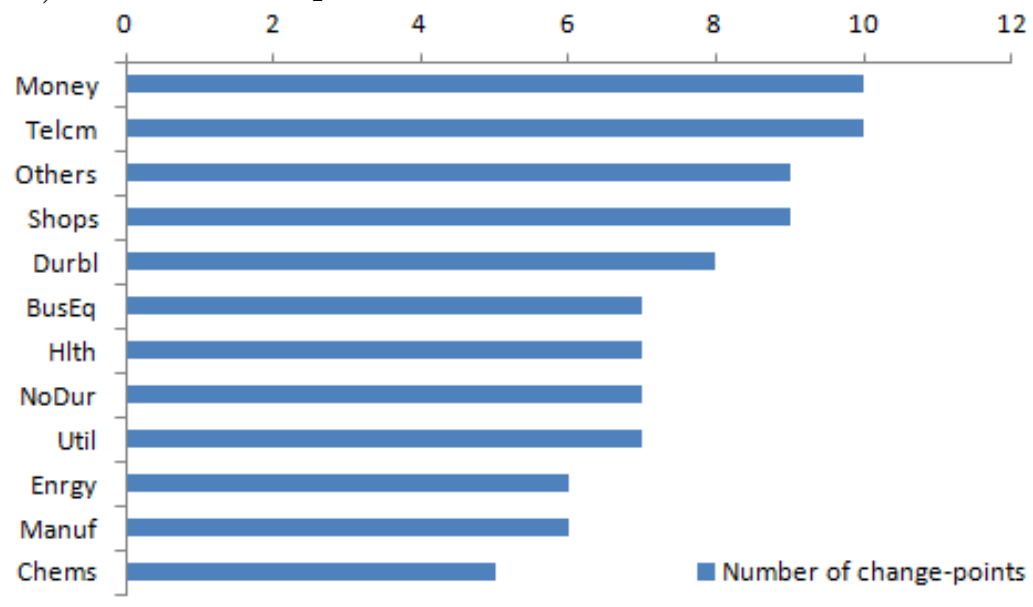


Figure 5: Sector co-crash change-points. Number of co-crash change-points ( $x$ axis) experienced by each sector ( $y$ axis) on the whole period.

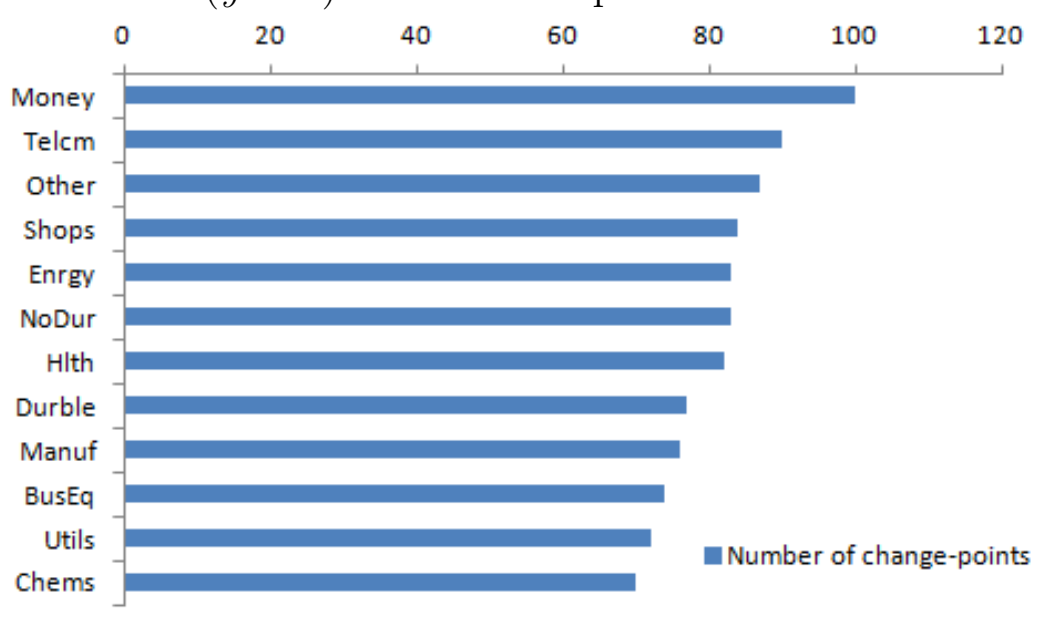

Figure 6: Number of co-crash change-points. Number of sectors-pair ( $x$ axis) experiencing a change-point in the co-crash probability in a given year ( $y$ axis).

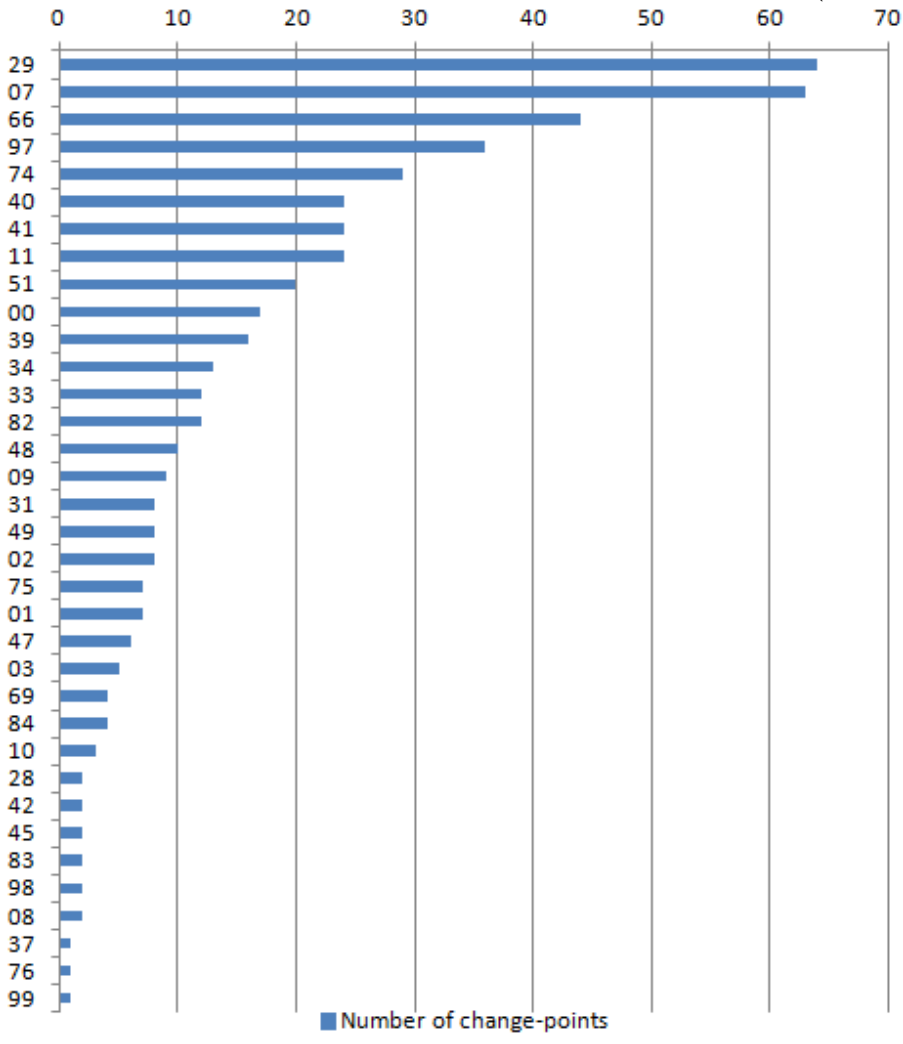


Table 3: Lower tail change-points dates.

\begin{tabular}{|c|c|c|c|c|}
\hline \multirow[b]{2}{*}{ Sectors } & \multicolumn{2}{|c|}{ Great Depression } & \multicolumn{2}{|c|}{ Great Recession } \\
\hline & Pre-crisis & Post-crisis & Pre-crisis & Post-crisis \\
\hline Business Equipment & 1929-10-02 & $1933-10-30$ & - & - \\
\hline Chemicals & 1929-09-26 & 1933-10-19 & $2007-07-25$ & - \\
\hline Durable & 1929-10-02 & 1939-09-18 & $2007-07-25$ & $2009-10-28$ \\
\hline Energy & 1929-10-02 & 1941-02-14 & - & - \\
\hline Health & $1928-10-18$ & $1930-10-08$ & - & - \\
\hline Manufacturing & 1929-10-02 & 1931-09-12 & $2007-07-23$ & - \\
\hline Money & 1929-09-26 & $1934-05-10$ & 2007-07-09 & 2009-07-02 \\
\hline No Durable & 1929-10-02 & 1941-02-14 & $2007-07-25$ & - \\
\hline Other & 1928-12-05 & 1931-09-01 & $2007-10-18$ & 2011-12-08 \\
\hline Shops & $1929-10-18$ & $1934-07-26$ & $2007-07-23$ & $2010-08-11$ \\
\hline Telecommunications & $1929-07-27$ & 1931-09-23 & 2007-10-31 & - \\
\hline Utilities & 1929-10-02 & 1939-09-18 & $2007-05-23$ & 2009-07-02 \\
\hline
\end{tabular}

Table 4: Upper tail GP parameters.

\begin{tabular}{|c|c|c|c|c|c|c|c|c|}
\hline \multirow[b]{3}{*}{ Sectors } & \multicolumn{4}{|c|}{ Great Depression } & \multicolumn{4}{|c|}{ Great Recession } \\
\hline & \multicolumn{2}{|c|}{ Pre-shock } & \multicolumn{2}{|c|}{ Post-shock } & \multicolumn{2}{|c|}{ Pre-shock } & \multicolumn{2}{|c|}{ Post-shock } \\
\hline & $\nu$ & $\xi$ & $\nu$ & $\xi$ & $\nu$ & $\xi$ & $\nu$ & $\xi$ \\
\hline Business Equipment & 0.73 & 0.52 & 1.66 & 0.22 & 0.47 & -0.16 & 1.43 & -0.11 \\
\hline Chemicals & 0.60 & 0.83 & 1.64 & 0.18 & 0.60 & -0.04 & 1.32 & 0.10 \\
\hline Durable & 1.04 & 0.32 & 1.45 & 0.39 & 0.54 & 0.04 & 1.86 & -0.06 \\
\hline Energy & 0.32 & -0.01 & 1.19 & 0.14 & 0.64 & 0.07 & 2.34 & 0.01 \\
\hline Health & 0.48 & 0.29 & 1.92 & 0.33 & 0.35 & -0.01 & 1.00 & 0.01 \\
\hline Manufacturing & 0.34 & -0.04 & 1.49 & 0.28 & 0.38 & 0.20 & 1.62 & -0.03 \\
\hline Money & 0.92 & 0.42 & 2.24 & 0.17 & 0.35 & 0.05 & 1.60 & -0.03 \\
\hline No Durable & 0.37 & 0.80 & 1.31 & 0.28 & 0.17 & 0.14 & 1.36 & -0.12 \\
\hline Other & 0.75 & 0.26 & 2.10 & 0.12 & 0.38 & 0.23 & 1.31 & -0.10 \\
\hline Shops & 0.35 & 0.09 & 1.23 & 0.21 & 0.27 & 0.13 & 1.43 & -0.11 \\
\hline Telecommunications & 0.83 & -0.12 & 1.19 & -0.01 & 0.50 & -0.36 & 1.43 & 0.04 \\
\hline Utilities & 0.42 & 0.08 & 0.95 & 0.42 & 0.34 & 0.32 & 1.19 & 0.41 \\
\hline
\end{tabular}


Table 5: Lower tail GP parameters.

\begin{tabular}{|c|c|c|c|c|c|c|c|c|}
\hline \multirow[b]{3}{*}{ Sectors } & \multicolumn{4}{|c|}{ Great Depression } & \multicolumn{4}{|c|}{ Great Recession } \\
\hline & \multicolumn{2}{|c|}{ Pre-shock } & \multicolumn{2}{|c|}{ Post-shock } & \multicolumn{2}{|c|}{ Pre-shock } & \multicolumn{2}{|c|}{ Post-shock } \\
\hline & $\nu$ & $\xi$ & $\nu$ & $\xi$ & $\nu$ & $\xi$ & $\nu$ & $\xi$ \\
\hline Business Equipment & 0.95 & -0.06 & 2.06 & 0.12 & - & - & - & - \\
\hline Chemicals & 0.92 & -0.12 & 1.43 & 0.33 & 0.35 & 0.20 & 1.55 & -0.01 \\
\hline Durable & 1.10 & -0.06 & 1.71 & 0.13 & 0.59 & 0.14 & 2.19 & -0.22 \\
\hline Energy & 0.61 & 0.08 & 1.36 & 0.12 & - & - & - & - \\
\hline Health & 0.60 & 0.75 & 1.25 & 0.23 & - & - & - & - \\
\hline Manufacturing & 0.79 & 0.01 & 1.64 & 0.24 & 0.44 & 0.12 & 1.61 & 0.02 \\
\hline Money & 0.41 & 0.50 & 1.54 & 0.20 & 0.31 & 0.35 & 1.88 & -0.21 \\
\hline No Durable & 0.64 & -0.04 & 0.93 & 0.32 & 0.36 & 0.30 & 1.27 & -0.02 \\
\hline Other & 0.60 & -0.14 & 1.01 & 0.25 & 0.48 & 0.21 & 1.41 & -0.05 \\
\hline Shops & 0.56 & 0.07 & 1.23 & 0.21 & 0.41 & 0.35 & 1.31 & 0.04 \\
\hline Telecommunications & 0.32 & 0.26 & 0.92 & 0.27 & 0.71 & -0.10 & 1.32 & 0.09 \\
\hline Utilities & 0.56 & 0.12 & 1.53 & 0.06 & 0.45 & 0.24 & 1.67 & -0.09 \\
\hline
\end{tabular}

\subsection{Co-crash and co-boom change-points}

We now turn our attention to the multivariate analysis performed with the algorithm outlined in Section 2.3. This allows us to understand the degree of contagion during the two crises, as measured by change-points leading to a jump in the co-crash probability. Tables 6 and 7 report the change-points in $\eta_{U P}$ and $\eta_{L O W}$ respectively for the Great Depression and the Great Recession.

First of all, in both crises the joint probability of a crash changes in all economic sectors. From Figure 6 we can say that this can be considered a peculiarity of these two crises. Furthermore, Figure 7 highlights that the change in the co-crash probability is upward, implying an increase of systemic risk. Figure 5 confirms once again the leading role of the financial sector in the economy.

The main difference between the two crises is in the pattern of diffusion. Indeed, the Great Depression spreads the risk more rapidly, as all the change-points in the joint lower tail occur between September and October 1929. Contrarily, the Great Recession is a slower process with the first change-points occurring in July and October 2007. This is consistent 
with the different origins of the crises. The Great Depression started as a consequence of a sudden and fast slowdown of the stock market rally. The Great Recession started with the cooling of the Housing Market and this slowly affected the banking system and the economy as a whole.

Table 6: Great Depression co-crash and co-boom change-points. The upper triangle of the matrix reports the change-point dates $(\mathrm{mm}-\mathrm{yy})$ on the co-boom parameter $\eta_{U P}$. The lower triangle reports the change-point dates (mm-yy) on the co-crash parameter $\eta_{L O W}$.

\begin{tabular}{|c|c|c|c|c|c|c|c|c|c|c|c|c|}
\hline Sectors & $\beta^{3)^{0}}$ & $\sigma^{v^{5}}$ & $p^{\text {sे }}$ & स्ञ & 䟚 & si & $\sum^{0^{2}}$ & $\overbrace{}^{2}$ & $0^{x^{v^{v}}}$ & $\mathrm{c}^{\mathrm{N}^{Q^{5}}}$ & $\overbrace{}^{e^{E}}$ & $\omega^{2}$ \\
\hline BusEq & & $10-30$ & $10-29$ & 09-30 & 09-30 & 09-30 & $10-29$ & 09-30 & 09-30 & $10-29$ & $02-29$ & $10-29$ \\
\hline Chems & 09-29 & & $10-29$ & $10-30$ & $10-30$ & $11-30$ & $10-30$ & $11-30$ & $10-30$ & $12-30$ & $02-29$ & $10-30$ \\
\hline Durbl & $10-29$ & $10-29$ & & $11-30$ & 09-30 & 06-31 & 09-30 & 09-30 & 09-30 & $12-30$ & $02-29$ & $10-29$ \\
\hline Enrgy & $10-29$ & $10-29$ & $10-29$ & & 09-30 & 09-30 & 09-30 & $12-30$ & 09-30 & $12-30$ & $07-29$ & 09-30 \\
\hline Hlth & 09-29 & 09-29 & 09-29 & $10-29$ & & $11-30$ & 09-30 & 09-30 & 09-30 & 09-30 & $12-30$ & 02-31 \\
\hline Manuf & $10-29$ & $10-29$ & $10-29$ & $10-29$ & $10-29$ & & 09-30 & $06-31$ & $06-31$ & $12-30$ & $02-29$ & 06-31 \\
\hline Money & 09-29 & 09-29 & $10-29$ & $10-29$ & 09-29 & $10-29$ & & 09-30 & 06-31 & $12-30$ & $07-29$ & $10-29$ \\
\hline NoDur & $10-29$ & $10-29$ & $10-29$ & $10-29$ & $10-29$ & $10-29$ & $10-29$ & & 09-30 & $12-30$ & $02-29$ & 04-31 \\
\hline Other & $10-29$ & $10-29$ & 09-29 & $10-29$ & 09-29 & 09-29 & $10-29$ & 09-29 & & $10-29$ & $07-29$ & 06-31 \\
\hline Shops & $10-29$ & $10-29$ & $10-29$ & $10-29$ & 09-29 & $10-29$ & $10-29$ & $10-29$ & 09-29 & & $03-29$ & $10-29$ \\
\hline Telcm & 09-29 & 09-29 & 09-29 & $10-29$ & $11-29$ & 09-29 & 09-29 & $10-29$ & $11-29$ & 09-29 & & 06-29 \\
\hline Util & $10-29$ & $10-29$ & $10-29$ & $10-29$ & $10-29$ & $10-29$ & $10-29$ & 09-29 & $10-29$ & $10-29$ & $07-29$ & \\
\hline
\end{tabular}

\section{Real-time monitoring}

In the previous sections, we perform a comparative analysis of the crises that hit the economy in the last century. Since our change-points algorithm can be only used a posteriori, it gives insights on the changes that occurred in the tail behaviour of the sectors we considered, but does not say anything about the possibility of forecasting periods of turmoil. We now question whether the test in Equation (5) can be used for real-time monitoring of the economy and to possibly signal whether tail risk is increasing.

We put ourselves in the shoes of a group of economists with the task of assessing the risk of a crisis at the beginning of 2007. How can we use the available test statistic and 
Table 7: Great Recession co-crash and co-boom change-points. The upper triangle of the matrix reports the change-point dates (mm-yy) on the co-boom parameter $\eta_{U P}$. The lower triangle reports the change-point dates $(\mathrm{mm}-\mathrm{yy})$ on the co-crash parameter $\eta_{L O W}$.

\begin{tabular}{|c|c|c|c|c|c|c|c|c|c|c|c|c|}
\hline Sectors & $s^{30}$ & $0^{v^{8 v}}$ & $\nabla^{\circledR}$ & से & 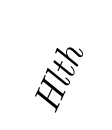 & ș & $\sum^{0^{2}}$ & $\overbrace{}^{2}$ & $0^{x^{c}}$ & 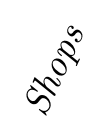 & $\hat{v}^{e^{\delta}}$ & $\omega^{\infty}$ \\
\hline BusEq & & 03-08 & $02-08$ & 09-08 & 09-08 & 09-08 & 03-08 & 09-08 & 09-08 & 09-08 & 09-08 & 03-08 \\
\hline Chems & $10-07$ & & $11-07$ & 01-08 & 03-08 & $11-07$ & $11-07$ & $11-07$ & $11-07$ & $11-07$ & $07-08$ & $11-07$ \\
\hline Durbl & $10-07$ & $07-07$ & & 09-08 & 06-08 & $11-07$ & $11-07$ & $11-07$ & $11-07$ & $11-07$ & 01-08 & $11-07$ \\
\hline Enrgy & $10-07$ & $10-07$ & $07-07$ & & 09-08 & 01-08 & 08-07 & 01-08 & 09-08 & 01-08 & 09-08 & $08-07$ \\
\hline Hlth & $10-07$ & $10-07$ & $10-07$ & $10-07$ & & 03-08 & 07-08 & 07-08 & $07-08$ & 03-08 & 06-08 & 09-08 \\
\hline Manuf & $10-07$ & $07-07$ & $07-07$ & $07-07$ & $10-07$ & & 08-07 & $11-07$ & $11-07$ & $11-07$ & 01-08 & $08-07$ \\
\hline Money & $07-07$ & $07-07$ & $02-07$ & $02-07$ & $02-07$ & $07-07$ & & $11-07$ & 08-07 & 08-07 & 08-07 & 08-07 \\
\hline NoDur & $10-07$ & $07-07$ & $07-07$ & $07-07$ & $10-07$ & $07-07$ & $07-07$ & & $11-07$ & $11-07$ & 01-08 & 01-08 \\
\hline Other & $10-07$ & $07-07$ & $07-07$ & $07-07$ & $10-07$ & $07-07$ & $07-07$ & $07-07$ & & $11-07$ & 03-08 & 08-07 \\
\hline Shops & $10-07$ & $07-07$ & $07-07$ & $07-07$ & $10-07$ & $07-07$ & $07-07$ & $07-07$ & $07-07$ & & 03-08 & $08-07$ \\
\hline Telcm & $10-07$ & $07-07$ & $07-07$ & $07-07$ & 08-08 & $07-07$ & $02-07$ & 07-07 & $07-07$ & $07-07$ & & 08-07 \\
\hline Util & $10-07$ & 07-07 & $07-07$ & 02-07 & 09-08 & $07-07$ & $07-07$ & $07-07$ & $07-07$ & $07-07$ & $07-07$ & \\
\hline
\end{tabular}

Figure 7: Co-crash index. Estimates of the tail dependence coefficient $\eta$ for each pair of economic sectors.

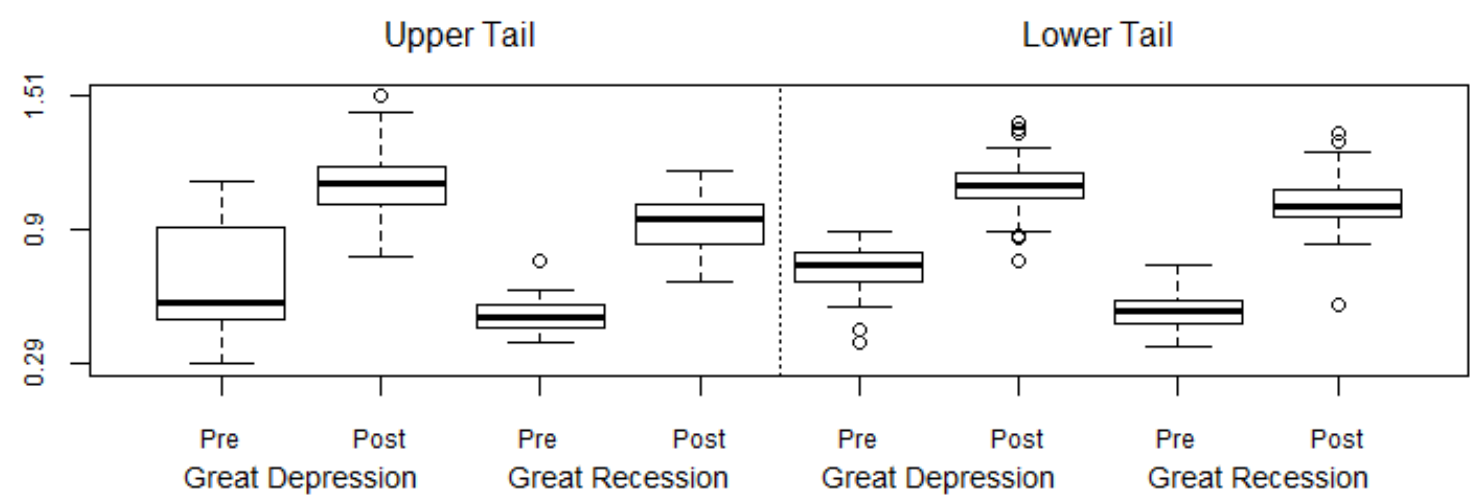


the market data on the US sectors for this purpose? We consider the last $S=1000$ days, hence the data from the beginning of 2003 to the beginning of 2007 and compute the test statistics in Equation (5). We choose this sample size because we need a sample large enough to compute the extreme value statistic, but at the same time we want the data to belong to the same economic regime. We retain the maximum value of $T_{n}$ within the last $\kappa=\{100,200,300,400\}$ days $^{1}$. We consider different values of $\kappa$ to trade-off the power of the test with the noise induced by including more past observations. We interpret a retained value exceeding the test critical value as a signal of changing tail behaviour, thus prompting a possible incumbent crises.

For each sector, we repeat this procedure every day till the end of 2008 in a rolling window fashion and display the results in Figure 8. We can see that for most of the series, a changing tail behaviour was evident before the end of 2007, close to the peak reached by the market on the 11th October 2007. Although we consider this date as the beginning of the crisis, we can see from the return time series in Figure 8 that the market did not panic until September 2008 where we can clearly observe a volatility burst in the data. Therefore, our test statistic signals an incumbent crisis well before the market explosion, and this evidence is strongest on the financial sector.

\section{Conclusion}

The collapse of the subprime mortgage market in the U.S. in 2007 and ensuing financial instability have spurred renewed interest in the origin of large scale crises. Some have stressed similarities across countries and historical episodes while others have emphasized differences, both historical and as related to the specific mechanics of the shock triggering a crisis. However, we all recognize that the Great Recession is an extraordinary, regime switchtype, event. It is clearly different, worse, larger, than usual recessions. Thus something happened to make the crisis fundamentally different from the usual economic downturn.

\footnotetext{
${ }^{1}$ This has the same effect of changing the parameter $\lambda$ in $T_{n}$ and keep the last value produced.
} 
Figure 8: Real-time monitoring. The azure, green ,blue and red lines are the values retained from $T_{n}$ with $\kappa=\{100,200,300,400\}$ respectively. The horizontal black line is the critical value at the $5 \%$ significance. The grey line represents the daily returns.
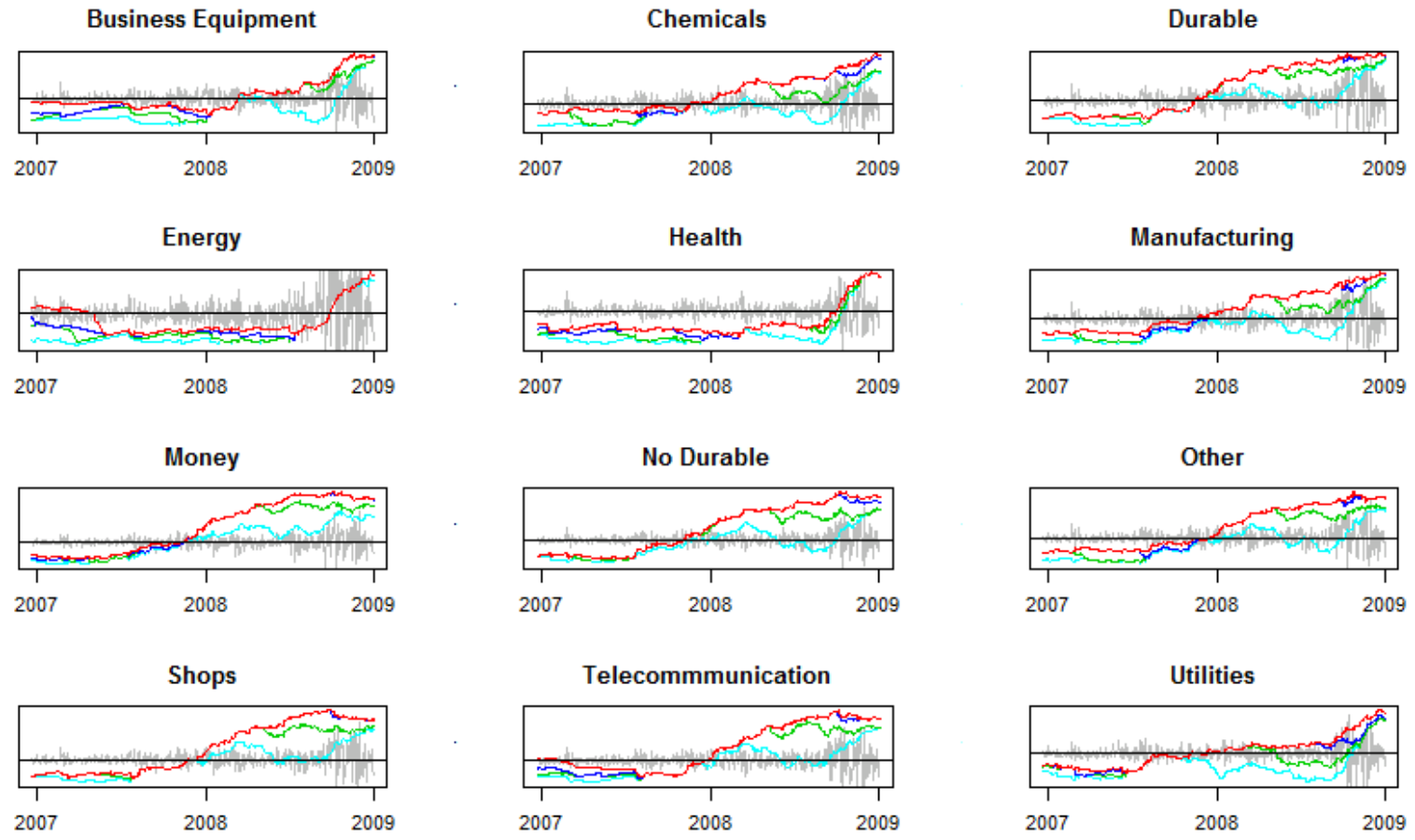
Historically, economic downturns that involve a financial crisis are worse than the usual downturns and stock market crashes are closely related to financial crises. Stock market regime switches do not happen at random times, but occur near the peak of the business cycle and after credit booms. This study adds to a recent strand of literature devoted to the comparison between major financial and economics crises of the last century, namely the Great Depression and the Great Recession. Unfortunately, the economic data available to study crises are usually scanty. Thus, contrarily to the mainstream papers focusing on the historical origins of the two crises and their macroeconomic aspects, we develop an empirical analysis of the U.S. economy based on market data obtained from the stocks traded on the main US stock markets. Since the largest financial crises are particularly likely to be accompanied by stock-market crashes, we analyze the spread of the crisis across different industries through the lens of Wall Street. Though limited in scope, our study provides interesting insights on the transmission mechanism between stock market collapse and economic recession. Furthermore, the availability of almost 100 years of stock market data allows for a full coverage of the two crises as well as other US business cycle contractions. This enable us to identify similarities and differences among major downturns.

We develop new procedures to detect change-points in the upper and lower tails of the asset return distribution and in the co-crash and co-boom probabilities between two assets. The iterative algorithms are fully automatic, therefore it is not necessary to specify in advance the dates of the change-points. The algorithms identify the change-points using only the information provided by the whole time series. Our methodology thus help in the dating of the start and the end of a crisis. In the literature the dating of crisis events differ quite significantly and there is also some disagreement on which events are crises. Further, typically the start dates are late since is mostly based on contemporary accounts of the governmental response to the crisis and not on the early effects of the crisis.

In our study we find that all the economic sectors present both a change-point around the crucial dates of the two crises and a change-point in the dependence structure. More- 
over, they tend to anticipate the stock market collapses. This evidence strongly suggests that both the Great Depression and the Great Recession carried a huge systemic risk and that the market uncertainty continued for several months before the crashes. Thus our methodological approach should be considered to update measurement systems to detect the buildup of systemic risk. In our work we identify the systemic effect of the crisis in the co-occurrence of a sudden negative shift in the tail risk of many sectors of the economy. This is a distinctive landmark of both the Great Depression and the Great Recession that deserves further scrutiny.

Even though, there are many things we do not know about the dynamics of crises, we hope our contribution helps in developing early warning mechanisms to detect the buildup of systemic risk in the stock market. As new data becomes available about the banking sector and other markets, in future work we plan to extend our approach to detect systemic risk in the probability of co-crash of both real and financial assets.

\section{References}

Barro, R. J. and Ursúa, J. F. Stock-market crashes and depressions. Technical report, National Bureau of Economic Research, 2009.

Bee, M., Dupuis, D., and Trapin, L. U.S. stock returns: Are there season of excesses? Unpublished manuscript, 2015.

Bollerslev, T. Generalized autoregressive conditional heteroskedasticity. Journal of econometrics, 31(3):307-327, 1986.

Bordo, M. and James, H. The great depression analogy. Financial History Review, 17(02): $127-140,2010$.

Candelon, B. and Straetmans, S. Testing for multiple regimes in the tail behavior of emerging currency returns. Journal of International Money and Finance, 25(7):1187-1205, 2006. 
Carrasco, M. and Chen, X. Mixing and moment properties of various GARCH and stochastic volatility models. Econometric Theory, 18(01):17-39, 2002.

Drees, H. Weighted approximations of tail processes for $\beta$-mixing random variables. Annals of Applied Probability, 10(4):1274-1301, 2000.

Eggertsson, G. B. and Mehrotra, N. R. A model of secular stagnation. Technical report, National Bureau of Economic Research, 2014.

Embrechts, P., Klüppelberg, C., and Mikosch, T. Modelling Extremal Events: for Insurance and Finance, volume 33. Springer Science \& Business Media, 1997.

Fama, E. The behavior of stock-market prices. Journal of Business, 38(1):34-105, 1965.

Hill, B. A simple general approach to inference about the tail of a distribution. Annals of Statistics, 3(5):1163-1174, 1975.

Kim, M., Lee, S., et al. Test for tail index change in stationary time series with Pareto-type marginal distribution. Bernoulli, 15(2):325-356, 2009.

Ledford, A. W. and Tawn, J. A. Statistics for near independence in multivariate extreme values. Biometrika, 83(1):169-187, 1996.

Mandelbrot, B. The variation of certain speculative prices. Journal of Business, 36(4): 394-419, 1963.

Mikosch, T. and Starica, C. Limit theory for the sample autocorrelations and extremes of a GARCH(1,1) process. Annals of Statistics, pages 1427-1451, 2000.

Pickands, J. Statistical inference using extreme order statistics. Annals of Statistics, 3(1): $119-131,1975$.

Quintos, C., Fan, Z., and Phillips, P. C. Structural change tests in tail behaviour and the asian crisis. The Review of Economic Studies, 68(3):633-663, 2001. 
Stiglitz, J. Toward a general theory of deep downturns. Technical report, National Bureau of Economic Research, 2015.

Straetmans, S. T., Verschoor, W. F., and Wolff, C. C. Extreme US stock market fluctuations in the wake of 9/11. Journal of Applied Econometrics, 23(1):17-42, 2008. 


\section{Institution Markets \\ IMT}

INSTITUTE FOR ADVANCED STUDIES LUCCA

2016 ( ) IMT Institute for Advanced Studies, Lucca Piazza San ponziano 6, 5100 Lucca, Italy. www.imtlucca.it 